\title{
Primer registro del cangrejo verde, Carcinus maenas (Linnaeus, 1758), en Golfo Nuevo, Argentina: un nuevo límite norte de distribución en costas patagónicas
}

\author{
Pablo Javier Torres ${ }^{1,0}$ \& Ximena González-Pisani ${ }^{2}$ \\ ${ }^{1}$ Instituto de Biología Subtropical (IBS) (CONICET-UNaM), Posadas, Misiones, Argentina. ${ }^{2}$ Centro Nacional Patagónico \\ (CENPAT-CONICET), Puerto Madryn, Chubut, Argentina
}

\begin{abstract}
RESUMEN. El objetivo de esta nota es reportar la presencia del cangrejo verde europeo, Carcinus maenas en el Golfo Nuevo, en Chubut, Argentina. Este hallazgo extiende su rango de distribución $250 \mathrm{~km}$ al norte del primer reporte en el Golfo San Jorge, en las costas patagónicas. C. maenas es una de las especies invasoras acuáticas más exitosas del mundo, con poblaciones establecidas en todos los continentes. Es un depredador voraz, por lo que su presencia representa una amenaza para la integridad de los ecosistemas costeros del sur de Sudamérica y para las economías de las comunidades pesqueras de la región.
\end{abstract}

[Palabras clave: especie invasora, Golfo San Jorge, Puerto Madryn, Chubut, Argentina]

\begin{abstract}
AвSTRACT.First record of green crabs, Carcinus maenas (Linnaeus, 1758) in Golfo Nuevo, Chubut Argentina: a new northern limit of distribution in the Patagonian coast. This note was aimed at reporting the presence of the "European green crab" Carcinus maenas in the coast of Nuevo Gulf (Chubut, Argentina). This new record extends the actual distribution range of this species $250 \mathrm{~km}$ to the north of San Jorge Gulf, where it was previously reported. C. maenas is one of the most successful aquatic invaders in the world, with populations established on all continents, and represents a threat to the integrity of coastal ecosystems of southern South America, and also to the economies of fishing communities of South Atlantic.
\end{abstract}

[Keywords: invasive species, Golfo San Jorge, Puerto Madryn, Patagonia, Chubut]

\section{INTRODUCCIÓN}

El cangrejo verde europeo, Carcinus maenas, es considerada una de las 100 especies invasoras más peligrosas del mundo (Lowe et al. 2004) y produce efectos negativos en los ecosistemas marinos en diferentes partes del mundo. Compite con especies de cangrejos nativos y depreda recursos pesqueros como almejas, mejillones y otras especies en entornos naturales y en la acuicultura (Grosholz and Ruiz 1996; Yamada and Gillespie 2008; Weis 2010). C. maenas es nativo de Mauritania, en África noroccidental, y se ha expandido a través del Atlántico hasta el norte de Europa, Noruega e Islandia (Yamada and Gillespie 2008; Compton et al. 2010). En su área de distribución nativa, $C$. maenas se encuentra en sustratos duros y blandos de lugares protegidos y moderadamente expuestos al oleaje (Grosholz and Ruiz 1996). Esta especie es euritérmica; soporta temperaturas inferiores a $0{ }^{\circ} \mathrm{C}$ y puede permanecer entre 31.3 y $35.8^{\circ} \mathrm{C}$ durante períodos breves, aunque el desarrollo de embriones y larvas sólo es completado por encima de $10^{\circ} \mathrm{C}$ y por debajo de un rango entre

Editor asociado: David Bilenca

$\triangle$ javier_codium@hotmail.com
18 y $26^{\circ} \mathrm{C}$ (Cohen et al. 1995; Cuculescu et al. 1998; Jamieson et al. 2002). Por otra parte, es capaz de resistir salinidades tan bajas como $3.17 \mathrm{~g} / \mathrm{L}$ en las etapas larvarias y $4 \mathrm{~g} / \mathrm{L}$ en las etapas adultas (Jamieson et al. 2002).

C. maenas se ha establecido en Sudáfrica, este de Australia, Tasmania, las costas atlántica y pacífica de América del Norte y Japón (Cohen et al. 1995; Compton et al. 2010). Carlton and Cohen (2003), basados en análisis de promedios de temperatura superficial del agua de mar, pronosticaron que la Patagonia estaría expuesta a la invasión por parte de esta especie. En la costa patagónica, Vinuesa (2005) registró el cangrejo verde europeo en la bahía de Camarones ( $\left.44^{\circ} 79^{\prime} \mathrm{S}\right)$, en Comodoro Rivadavia ( $45^{\circ} 52^{\prime}$ S) y en Rada Tily $\left(45^{\circ} 93^{\prime}\right.$ S) desde 2001 (Figura 1), e Hidalgo et al. (2005) lo registraron en bahía Camarones en noviembre de 2003 y enero de 2004. Además, Hidalgo et al. (2005) sugieren que el cangrejo podría colonizar la costa oriental de América del Sur desde el sur de Brasil ( $29^{\circ} \mathrm{S}$ ) hasta la boca del Estrecho de Magallanes (52 ${ }^{\circ}$ S) (Figura 1). 


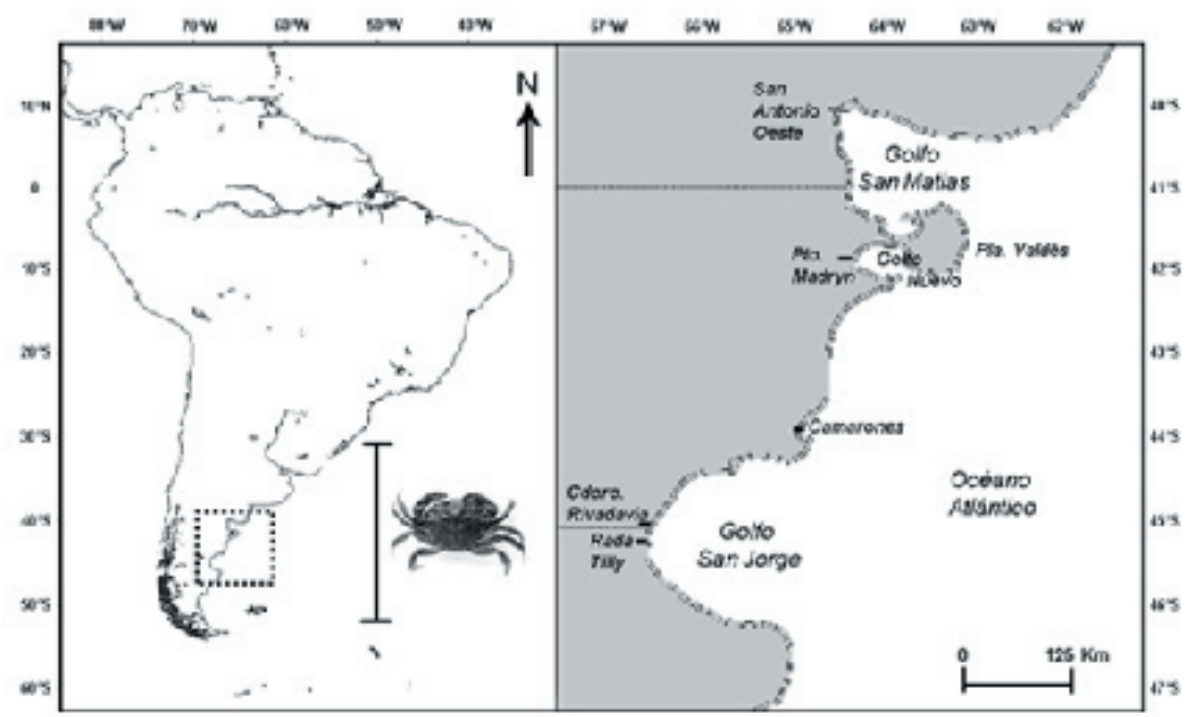

Figura 1. a) Distribución potencial de C. maenas en la costa este de América del Sur, según Hidalgo et al. (2005); distribución actual de $C$. maenas en cuadro de línea llena. b) Hallazgo de $C$. maenas en el Golfo Nuevo, según se indica en el presente trabajo; Bahía de Camarones, Ciudad de Comodoro Rivadavia, Rada Tilly y Puerto Madryn.

Figure 1. a) Potential distribution of C. maenas in the eastern coast of South America, according to Hidalgo et al. (2005). b) Finding of $C$. maenas in Nuevo Gulf, as indicated by the current study; Bay Camarones, City of Comodoro Rivadavia, Rada Tilly and City of Puerto Madryn.

\section{MATERIALES y MÉTODOS}

En marzo de 2015, se recolectaron cangrejos de forma recreativa mediante inmersiones en apnea en la costa del Golfo Nuevo, en el intermareal rocoso situado entre dos puertos marítimos internacionales de la ciudad de Puerto Madryn ( $\left.42^{\circ} 46^{\prime} \mathrm{S}-65^{\circ} 02^{\prime} \mathrm{O}\right)$ Argentina (Figura 1b). Uno de ellos, "Almirante Storni", opera el transporte de minerales, y el otro, "Luis Piedra Buena", es utilizado para la pesca y el turismo. La zona recorrida es una plataforma de abrasión cubiertas por mejillones, con pozas de marea, rocas y sedimentos arenosos (Figura 2a). Los especímenes se identificaron en laboratorio como pertenecientes a Carcinus maenas, siguiendo los caracteres taxonómicos reportados por Yamada and Hauck (2001), donde mencionan como principales caracteres diagnósticos la forma de los pleopodos de los machos (tocándose en sus centros y separándose en los extremos), la parte frontal entre los ojos (lobulada) y la relación de ancho de caparazón (AC) y largo de caparazón (LC), $A C / L C>1.29$. Además, fueron comparados con ejemplares de la misma especie colectados en la bahía Camarones por los autores de este trabajo. Los ejemplares fueron sexados y medidos con un calibre de $0.01 \mathrm{~mm}$ de precisión. Las medidas tomadas fueron el ancho máximo de caparazón entre las espinas laterales y, en el caso de los machos, largo máximo del artejo fijo que conforma la quela.

\section{RESULTADOS Y DiscusióN}

Se han encontrado individuos (macho) de Carcinus maenas $(\mathrm{n}=10)$ en la plataforma de abrasión cubierta por mejillones (Mytilus sp.) (Figura 2a), con pozas de marea, rocas y en sedimentos arenosos. El ancho del caparazón de los individuos capturados osciló entre 37.8 y $89.5 \mathrm{~mm}$ (media $=63.65 \pm 36.57 \mathrm{~mm}$ ) (Figura 2b). Los individuos encontrados son similares en tamaño a los reportados para Estados Unidos y más grandes que los hallados en Sudáfrica y Europa (Grosholz and Ruiz 1996). Sin embargo, Hidalgo et al. (2005) reportaron un menor rango de tamaños en la población de machos de la bahía Camarones (39.9-71.4 mm de ancho de caparazón). Dadas las tallas máximas registradas en la muestra de la ciudad de Puerto Madryn, se estima que son individuos adultos, reproductivamente aptos. Hasta 2014 se había reportado la presencia de 29 especies exóticas en el Golfo Nuevo (Schwindt et al. 2014). Dos de ellas son las especies invasoras más importantes, responsables de cambios sustanciales en los ecosistemas marinos del Golfo Nuevo. Una es el cirripedio Balanus glandula, nativo de la costa del Pacífico de América del Norte, que además de colonizar sustratos de fondos blandos (atípicos para la especie) recluta en altas densidades, formando agregados tridimensionales y, por ende, nuevos hábitats para otras especies de invertebrados intermareales. Es un componente crítico 


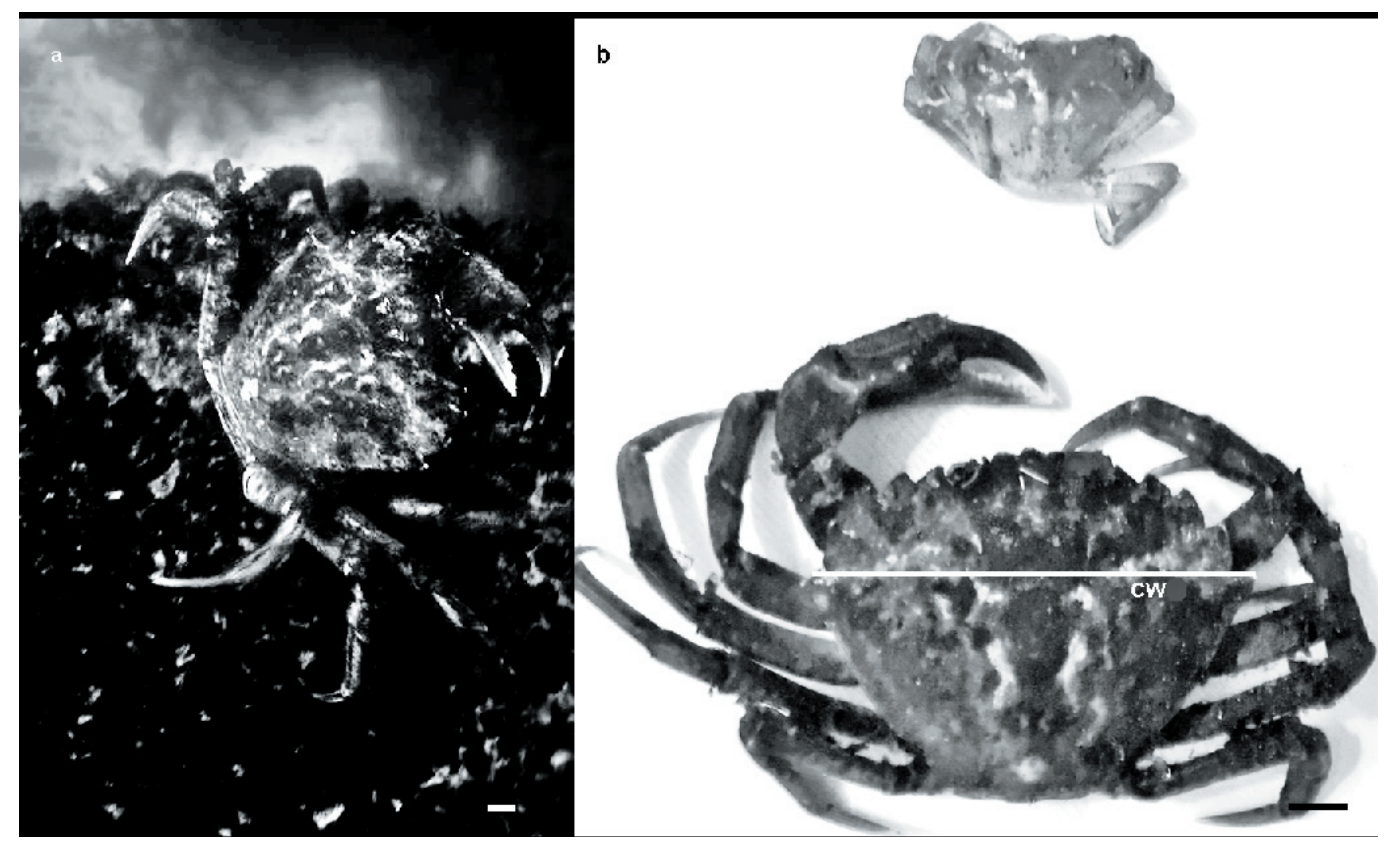

Figura 2. a) Vista de Carcinus maenas sobre rocas cubiertas por mejillones. b) Vista dorsal de Carcinus maenas. Arriba: el cangrejo más pequeño capturado (ancho de caparazón $(\mathrm{CW}): 37.8 \mathrm{~mm}$ ). Abajo: el cangrejo capturado de mayor tamaño (ancho de caparazón [CW]: $89.5 \mathrm{~mm}$ ). Escala: $1 \mathrm{~cm}$.

Figura 2. a) Carcinus maenas observed on rocks covered by mussels. b) Dorsal view of $C$ maenas. Above: the smallest crab caught (CW [carapace width]: $37.8 \mathrm{~mm}$ ). Below: the biggest crab caught (CW: $89.5 \mathrm{~mm})$. Scale: $1 \mathrm{~cm}$.

en la estructuración de la comunidad de invertebrados nativos (Schwindt 2007; Méndez et al. 2015). La segunda es la macroalga Undaria pinnatifida, nativa de las costas de Japón, Corea y China, que se asocia a una disminución de la riqueza de especies y diversidad de algas nativas (Casas and Piriz 1996; Casas et al. 2004). Con el presente reporte se suma a dicha lista una especie más: el cangrejo verde Carcinus maenas, del cual cabe esperar un gran impacto sobre el ecosistema, a la vez que una reducción de la biodiversidad, ya sea por los efectos de la depredación, la competencia con otras especies y la modificación de las comunidades bentónicas (McDonald et al. 2001; Walton et al. 2002; Grosholz and Ruiz 1996). Lo dicho cobra particular relevancia teniendo en cuenta que en la zona adyacente al sitio de hallazgo de este nuevo invasor se encuentra la Península Valdés, área declarada como patrimonio de la humanidad por la Unesco en 1999. Esperamos que este hallazgo contribuya a diseñar programas de monitoreo más ajustados y una normativa adecuada para prevenir nuevas invasiones biológicas.

Agradecimientos. Los autores agradecen al Tec. Sup. Acuicultura Mariano Moris (CENPAT-CONICET) por su ayuda en la toma de muestras, al Dr. Gaspar Soria (CENPATCONICET) por sus útiles comentarios, a la Srta. Jimena Villahoz por la revisión del lenguaje y a la Srta. Agustina Canale por la edición de fotografía.

\section{REFERENCIAS}

Carlton, J. T., and A. N. Cohen. 2003. Episodic global dispersal in shallow water marine organisms: the case history of the European shore crabs Carcinus maenas and Carcinus aestuarii. J Biogeogr 30:1809-1820. doi: 10.1111/j.13652699.2003.00962.x.

Casas, G. M., and M. L. Piriz. 1996. Surveys of Undaria pinnatifida (Laminariales, Phaeophyta) in Golfo Nuevo, Argentina. Hydrobiologia 327:213-215. doi: 10.1007/978-94-009-1659-3_29.

Casas, G., R. Scorsati, and M. L. Piriz. 2004. The invasive kelp Undaria pinnati4da (Phaeophyceae, Laminariales) reduces native seaweed diversity in Nuevo Gulf (Patagonia, Argentina). Biological invasions 6:411-416.

Cohen, A. N., J. T. Carlton, and M. Fountain. 1995. Introduction, dispersal and potential impacts of the green crab Carcinus maenas in San Francisco Bay, California. Mar Biol 122:225-237. doi: 10.1007/BF00348935

Compton, T. J., I. R. Leathwick, and G. J. Inglis. 2010. Thermogeography predicts the potential global range of the invasive European green crab (Carcinus maenas). Diversity Distrib 16:243-255. doi: 10.1111/j.1472-4642.2010.00644.x.

Cuculescu, M., D. Hyde, and K. Bowler. 1998. Thermal tolerances of two species of marine crabs, Cancer pagurus and 
Carcinus maenas. J Therm Biol 23(2):107-110.

Grosholz, E. D., and G. M. Ruiz. 1996. Predicting the impact of introduced marine species: lessons from the multiple invasions of the European green crab Carcinus maenas. Biol Conserv 78:59-66.

Hidalgo, F. J., P. J. Baron, and J. M. Orensanz. 2005. A prediction come true: the green crab invades the Patagonian coast. Biol Invasions 7:547-552.

Jamieson, C. S., M. G. G. Foreman, J. Y. Cherniawsky, and C. D. Levings. 2002. European green crab (Carcinus maenas) dispersal: the pacific experience. Pp. 561-576 in: A. J. Paul, E. G. Dawe, R. Elner, G. S. Jamieson, G. H. Kruse, R. S. Otto, B. Sainte-Marie, T. C. Shriley, and D. Eoodby (eds.). Crabs in Cold Water Regions: Biology, Management and Economics. Proceedings of the Symposium Sea Crab 2001. University of Alaska, Sea Grant College Program.

Lowe, S., M. Browne, S. Boudjelas, and M. De Poorter. 2004. 100 of the World's worst invasive alien species. A selection from the Global Invasive Species Database. The Invasive Species Specialist Group (ISSG) a specialist group of the Species Survival Commission (SSC) of the World Conservation Union (IUCN)

McDonald, A., L. Jensen, and A. Armstrong. 2001. The competitive and predictory impacts of the non-indigenous crab Carcinus maenas L. on early benthic phase Dungeness crab Cancer magister Dana. Journal of Experimental Marine Biology and Ecology 258:39-54.

Méndez, M. M., E. Schwindt, and A. Bortolus. 2015. Differential benthic community response to increased habitat complexity mediated by an invasive barnacle. Aquat Ecol Aquatic Ecology 49:441-452.

Schwindt, E. 2007. The invasion of the acorn barnacle Balanus glandula in the south-western Atlantic 40 years later. J Mar Biol Ass UK 87:1219-1225.

Schiwindt, E., J. L. Gappa, M. Raffo, M. Tatian, A. Bortolus, J. M. Orensanz, M. Alonso, M. Diez, B. Doti, G. Genzano, C. Lagger, G. Lovrich, M. Piriz, M. Méndez, V. Savoya, and M. Sueiro. 2014. Marine fouling invasions in ports of Patagonia (Argentina) with implications for legislation and monitoring programs. Marine Environmental Research 90:60-68.

Vinuesa, J. H. 2005. Distribución de crustáceos decápodos y estomatópodos del golfo San Jorge, Argentina. Rev Biol Mar Oceanogr 40:7-21.

Walton, W. C., C. MacKinnon, L. Rodríguez, C. Proctor, R. Thresher, and G. M. Ruiz. 2002. Effect of an invasive crab upon a marine fishery: green crab Carcinus maenas, predation upon a venerid clam Katelysia scalarina, in Tasmania (Australia). Journal of Experimental Marine Biology and Ecology 272:171-189.

Weis, J. S. 2010. The role of behavior in the success of invasive crustaceans. Mar Freshw Behav Physiol 43:83-98.

Yamada, S. B., and L. Hauck. 2001. Field identification of the European green crab species: Carcinus maenas and Carcinus aestuarii. J Shellfish Res 20:905-912.

Yamada, S. B., and G. E. Gillespie. 2008. Will the European green crab (Carcinus maenas) persist in the Pacific Northwest? ICES J Mar Sci: Journal du Conseil 65:725-729. 